\title{
Balancing inflammation and tolerance in vivo through dendritic cells by the commensal Candida albicans
}

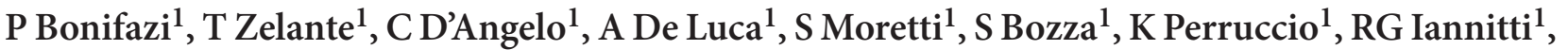 \\ G Giovannini $^{1}$, C Volpi ${ }^{1}$, F Fallarino ${ }^{1}$, P Puccetti $^{1}$ and L Romani ${ }^{1}$
}

\begin{abstract}
We analyzed the contribution of intracellular signaling to the functional plasticity of dendritic cells (DCs) presenting Candida albicans, a human commensal associated with severe diseases. Distinct intracellular pathways were activated by recognition of different fungal morphotypes in distinct DC subsets and in Peyer's patches DCs. Inflammatory DCs initiated Th17/Th2 responses to yeasts through the adaptor myeloid differentiation factor-88 (MyD88), whereas tolerogenic DCs activate Th1/T regulatory cell (Treg) differentiation programs to hyphae involving Toll/IL-1 receptor domain-containing adaptor inducing IFN-beta (TRIF) as an intermediary of signaling. In addition, signal transducer and activator of transcription 3 (STAT3), affecting the balance between canonical and non-canonical activation of nuclear factor-kappaB (NF-kB) and 2,3 indoleamine dioxygenase (IDO), pivotally contributed to DC plasticity and functional specialization. As Candida-induced tolerogenic DCs ameliorated experimental colitis, our data qualify Candida as a commensal with immunoregulatory activity, resulting from the orchestrated usage of multiple, yet functionally distinct, receptor-signaling pathways in DCs. Ultimately, affecting the local Th17/Treg balance might likely be exploited by the fungus for either commensalism or pathogenicity.
\end{abstract}

\section{INTRODUCTION}

Although associated with a number of severe diseases, Candida albicans is primarily a human commensal of mucosal and skin surfaces, a condition requiring a bidirectional interaction with the host immune system. Indeed, not only would the fungus benefit from a local anti-inflammatory/tolerogenic state for its own persistence, ${ }^{1}$ but through the modulation and induction of local regulatory $\mathrm{T}$ cells (Tregs) capable of dampening innate and adaptive immunity, ${ }^{2}$ C. albicans itself could also contribute to the maintenance of a tolerogenic state in the gut. However, the dual nature of commensal and pathogen would predict that the fungus would be able to modulate immune reactivity in opposite directions. As a matter of fact, besides infection, a number of dysregulated immune reactivities have been associated with the fungus and/or certain fungal components. ${ }^{3}$

The exploitation of distinct recognition receptors in dendritic cells (DCs) determines the full range of the host's immune relationships with C. albicans. ${ }^{4-6}$ Similar to what has been reported with mononuclear phagocytes, ${ }^{7,8}$ distinct intracellular signaling pathways are likely to play a role in the variegation of antifungal immune responses by DCs. Stimuli, including Toll-like receptor (TLR) agonists and endogenous molecules, differentially instruct DCs to initiate Th responses through the modulation of intracellular signaling pathways. ${ }^{9}$ A wealth of evidence indicates that DC immunogenicity/ tolerogenicity is not a characteristic of a specific subset or lineage of DCs, but an environmentally acquired feature. In this regard, the tryptophan metabolic pathway pivotally contributes to DC regulation, such that tolerance and Tregs induction could be mediated by DCs expressing the enzyme, 2,3 indoleamine dioxygenase (IDO). ${ }^{10,11}$

In this study, we sought to determine which molecular mechanisms underlie the plastic response of DCs to Candida and the functional consequences in terms of immune responses and immune homeostasis in vivo. We comparatively studied bone marrow-derived DCs, known for their specialization and complementarity in antifungal priming and tolerization in vivo ${ }^{11}$ and Peyer's patches (PP)-DCs, known for their immunoregulatory

1Department of Experimental Medicine and Biochemical Sciences, Microbiology Section, University of Perugia, Perugia, Italy. Correspondence: L Romani (Iromani@unipg.it) Received 3 December 2008; accepted 1 April 2009; published online 6 May 2009. doi:10.1038/mi.2009.17 


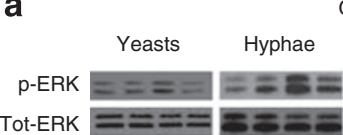

Tot-ERK $\equiv=\equiv=$ 플

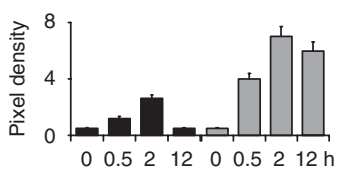

Yeasts

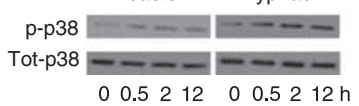

$00.521200 .5212 \mathrm{~h}$

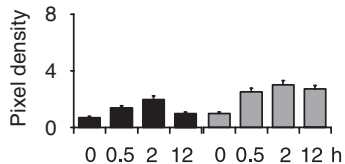

GM-DCs
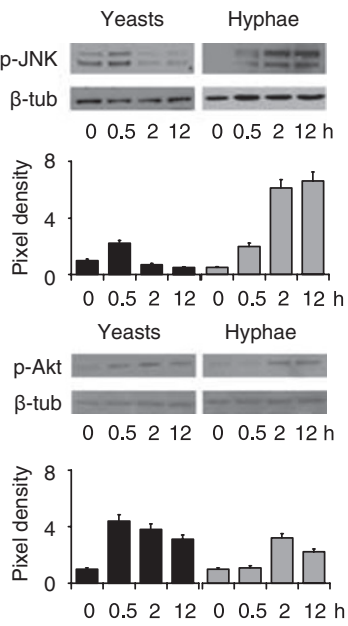

b FL-DCs

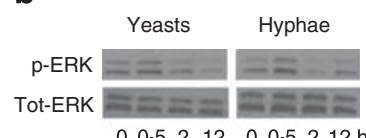

$00.521200 .5212 \mathrm{~h}$

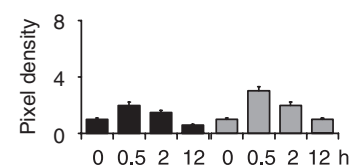

Yeasts Hyphae

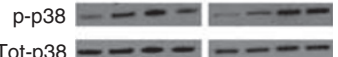

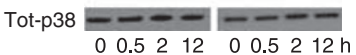

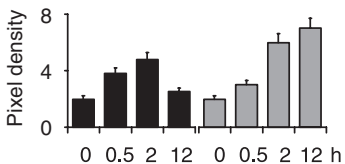

\section{C}

GM-DCs

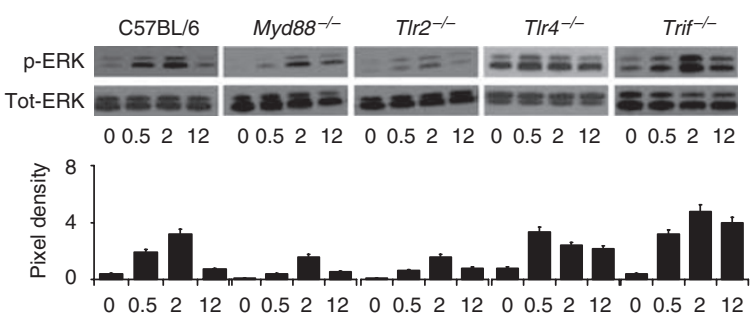

d
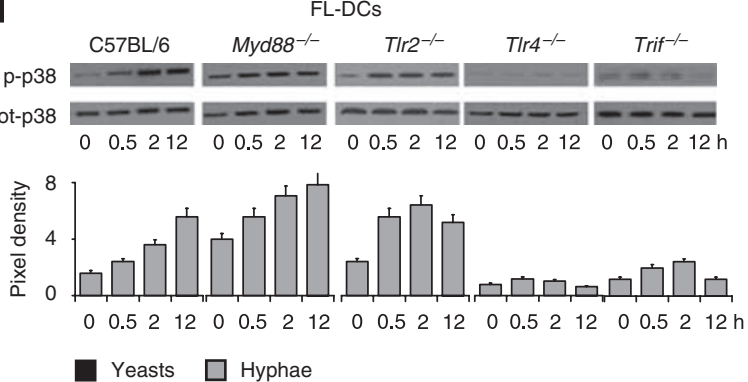

Figure 1 Activation of distinct intracellular kinases in dendritic cell (DC) subsets by Candida albicans. Levels of extracellular signal-regulated kinases (ERK1/2), Phospho SAPK/JNK (Thr183/Tyr185) (JNK1/2), p38 mitogen-activated protein kinase (MAPK) and Akt phosphorylation at different time points after stimulation of GM-CSF/L-4-derived (GM) or FLT3L-derived (FL) DCs from the bone marrow of (a, b) C57BL/6, (c, d) Toll-like receptor (TLR2), TLR4, myeloid differentiation factor-88 (MyD88), or Toll/IL-1 receptor domain-containing adaptor inducing IFN-beta (TRIF)-deficient mice with live unopsonized Candida yeasts (black histograms) or hyphae (gray histograms). Data are presented as immunoblots of cell lysates with phosphorylation-specific antibodies and fold increases (pixel density) in the phosphorylated to total protein ratios or to $\beta$-tubulin ( $\beta$-tub). Scanning densitometry was performed on a Scion Image apparatus. Immunoblots were reblotted for $\beta$-actin to ensure equal protein loading. The values are representative of five experiments.

function in mucosal candidiasis. ${ }^{12}$ We found that Candida exploits multiple, functionally distinct, receptor-signaling pathways in DCs ultimately affecting the local inflammatory/antiinflammatory state in the gut.

\section{RESULTS}

\section{Activation of distinct intracellular kinases in GM-DCs and FL-DCs}

The activation of intracellular mitogen-activated protein kinase (MAPK), extracellular signal-regulated kinases (ERK1/2), Jun $\mathrm{N}$-terminal kinases 1 and 2 (JNK 1/2), and p38, play a major role in DC function. ${ }^{13}$ We first performed a proteomic array analysis to simultaneously detect the relative level of phosphorylation of 19 kinases in DCs derived from murine bone marrow using either GM-CSF/IL-4 (GM-DCs) — which yields a subset of DCs very similar to CD8 $\alpha$-splenic inflammatory DC-or FLT3-L ( $m s$-like tyrosine kinase 3-ligand) (FL-DCs) - which is known to expand steady-state conventional $\mathrm{CD} 8^{-}, \mathrm{CD}^{+}$, and plasmacytoid (p)B220 ${ }^{+}$DCs. ${ }^{14}$ The results revealed that, $30 \mathrm{~min}$ after the exposure, Candida yeasts and hyphae triggered the phosphorylation of ERK1/2, JNK, and phosphoinositide 3-kinase (PI3K)-Akt in GM-DCs and the phosphorylation of p38 and glycogen synthase kinase- $3 \alpha / \beta$ in FL-DCs (Supplementary Figure S1). Assessed over time, there were differences in the 
magnitude and duration of ERK1/2, JNK, p38 MAPK, and Akt phosphorylation induced by the different stimuli. Consistent with the observation that hyphae survive longer than yeasts inside DCs, ${ }^{15}$ the activation of ERK1/2 and JNK in GM-DCs was prolonged $(2-12 \mathrm{~h})$ in response to hyphae and transient $(0.5-2 \mathrm{~h})$ in response to yeasts, and similar was $\mathrm{p} 38$ activation in FL-DCs in response to hyphae (Figure 1a and $\mathbf{b}$ ). Akt activation, by contrast, was increased early in response to yeasts but late in response to hyphae (Figure 1a). Confirming the proteomic analysis, the activations of ERK1/2 in FL-DCs and p38 in GMDCs in response to either of the stimuli were weak and transient (Figure 1a and b), whereas JNK and Akt signaling pathways were not detected in FL-DCs (data not shown).

We next assessed the relative contribution of myeloid differentiation factor-88 (MyD88) and Toll/IL-1 receptor domaincontaining adaptor inducing IFN-beta (TRIF), given their role in the functional maturation and specialization of murine bone marrow-derived DC subsets ${ }^{16}$ and upstream TLRs, known to play a role in the adaptive immune response to the fungus, ${ }^{2,8}$ on the activation of MAPK. ERK activation was decreased in the absence of TLR2 or MyD88 in GM-DCs, whereas p38 phosphorylation was reduced in the absence of TLR4 or TRIF in FL-DCs. In addition, the finding that either of the MAPKs was oppositely regulated by MyD88 and TRIF in the corresponding DC subset, i.e., increased ERK activation in the absence of TRIF and p38 activation in the absence of MyD88, suggests the occurrence of a reciprocal regulation between these two signaling pathways in DCs (Figure 1c and d). Similar to ERK, JNK, and Akt, phosphorylation was greatly increased in Trif $^{-1-}$ GM-DCs (data not shown), thus further suggesting that TRIF acts as a negative regulator of these signaling pathways in DCs.

\section{Activation of distinct nuclear factor-kappaB pathways in GM-DCs or FL-DCs}

Nuclear factor-kappaB (NF- $\kappa \mathrm{B})$ transcription factors are essential for regulating development, survival, and cytokine production by DCs. ${ }^{17} \mathrm{NF}-\kappa \mathrm{B}$ can be activated through two distinct signal transduction pathways, the canonical pathway culminating in DNA binding of the classical NF- $\kappa \mathrm{B}$ dimer, p50-RelA, and requiring IkappaB kinase-beta (IKK $\beta)$, or the non-canonical pathway that is strictly dependent on IkappaB kinase-alpha (IKK $\alpha$ ) and involves the proteolytic processing of p100 to p52. The two inhibitor of kappaB (IkB) kinase complex catalytic subunits have opposing roles in inflammation and immunity, with IKK $\alpha$ limiting the IKK $\beta$-dependent inflammatory pathway and leading to resolution of the early inflammatory process and development of Tregs. ${ }^{18}$ We examined DC subsets for a possible activation of either of the NF- $\kappa \mathrm{B}$ pathways in response to the fungus. The phosphorylation of IKK $\beta$, but not of IKK $\alpha$, was induced in GM-DCs in a MyD88-dependent manner; by contrast, both IKK $\beta$ and IKK $\alpha$ were induced in FL-DCs in a TRIF-dependent manner, particularly by hyphae (Figure 2a). Time course experiments showed that the activation of the canonical NF- $\kappa B$ pathway progressively increased in GM-DCs, as detected by the nuclear translocation of p65/p50 at $180 \mathrm{~min}$ after the exposure, but not in FL-DCs (Figure 2b), at the time at which the non-canonical pathway was instead activated (data not shown). This finding suggests that the canonical NF- $\kappa \mathrm{B}$ pathway is activated in GM-DCs through MyD88 by yeasts and hyphae, and the non-canonical pathway in FL-DCs by hyphae through TRIF.

\section{Induction of IDO in FL-DCs through the non-canonical NF- $\kappa B$ pathway}

The non-canonical NF- $\kappa$ B pathway is required for the expression of Indo, ${ }^{19,20}$ a suppressive mechanism of DCs. ${ }^{21,22}$ We investigated which NF- $\mathrm{KB}$ activation pathway mediates IDO protein expression in either of the DC subsets on blocking the canonical or non-canonical pathway by silencing IKK $\beta$ or IKK $\alpha$, respectively, with small interference RNA (siRNA). Confirming earlier data, ${ }^{2,19}$ IDO protein expression occurred in FL-DCs, but not in GM-DCs, in response to hyphae and was dependent on IKK $\alpha$ activation (Figure 2c). As a matter of fact, IDO appeared to be highly expressed by FL-DCs administered si-IKK $\beta$ in the absence of stimulation. These data, together with our earlier findings that showed that IDO expression in FL-DCs was TRIF-dependent but MyD88-independent, ${ }^{2}$ indicate that IDO activation in FLDCs occurs through the non-canonical NF- $\kappa \mathrm{B}$ pathway.

As IDO is induced in plasmacytoid dendritic cells ( $\mathrm{pDCs}$ ) through a TLR9/MyD88-dependent pathway, ${ }^{21}$ and FL-DCs encompass inflammatory as well as pDCs, we evaluate whether IDO induction in pDCs could account for IDO expression in FL-DCs. Contrary to CpG oligodeoxynucleotide (CpG-ODN), IDO was not induced in pDCs by the fungus, thus suggesting that Candida may not interact directly with pDCs for IDO protein induction. To gain insight into the possible mechanisms underlying IDO activation, we assessed immunoregulatory fungal pathogen-associated molecular pattern (PAMP), such as zymosan, glucan, and mannan, and defined TLR ligands for IDO induction in DCs. Similar to hyphae, the IDO expression was induced by lipopolysaccharide and zymosan, but not by glucan or mannan, in FL-DCs but not in GM-DCs or pDCs, a finding suggesting that the activation of IDO in FL-DCs is mediated by selected fungal ligands acting through TLR4 and TLR2 (Figure 2d).

\section{Signal transducer and activator of transcription 3 regulates the anti-inflammatory/regulatory state of DCs}

Signal transducer and activator of transcription 3 (STAT3) is indispensable in FL-DC development and ontogeny, conditions the tolerogenic state of DCs, and acts as a negative regulator of immunostimulatory DCs. ${ }^{23}$ As STAT3 activates the non-canonical NF- $\kappa B$ pathway while inhibiting the canonical NF- $\kappa B$ pathway, ${ }^{24}$ we assessed whether this would occur in response to fungi and the effect on IDO activation. We measured $\mathrm{Tyr}^{705}$ phosphorylation of STAT3, which promotes its transcription activity, in GM-DCs or FL-DCs and the dependency on MAPK, MyD88, and TRIF. STAT3 activation in GM-DCs did not occur before $12 \mathrm{~h}$ of stimulation with Candida yeasts (Figure 3a). By contrast, STAT3 activation in hypha-pulsed FL-DCs, whose basal levels of phosphorylation was notably higher compared with that of GMDCs, peaked at $3 \mathrm{~h}$, maintained elevated at $12 \mathrm{~h}$ (Figure 3a), and 
a
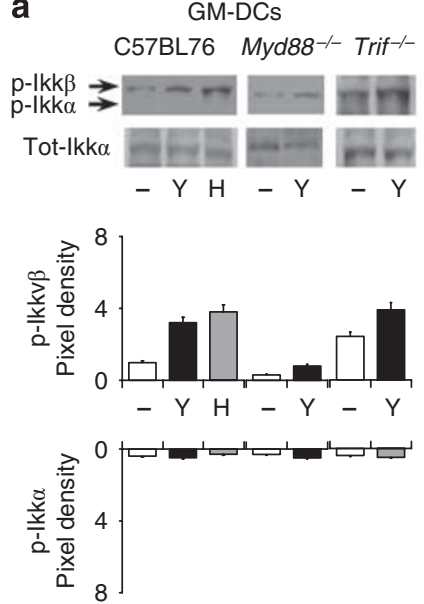

FL-DCs

C57BL/6 Myd88-/- Trif-/
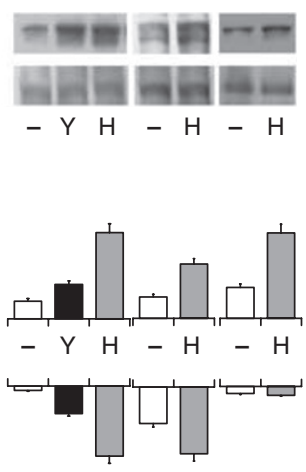

b
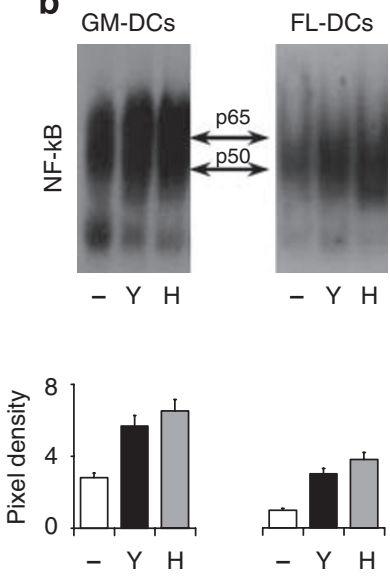

C

$M_{22} M_{24}$
IDO $=-$
$\beta$-tub $=-$

GM-DCs
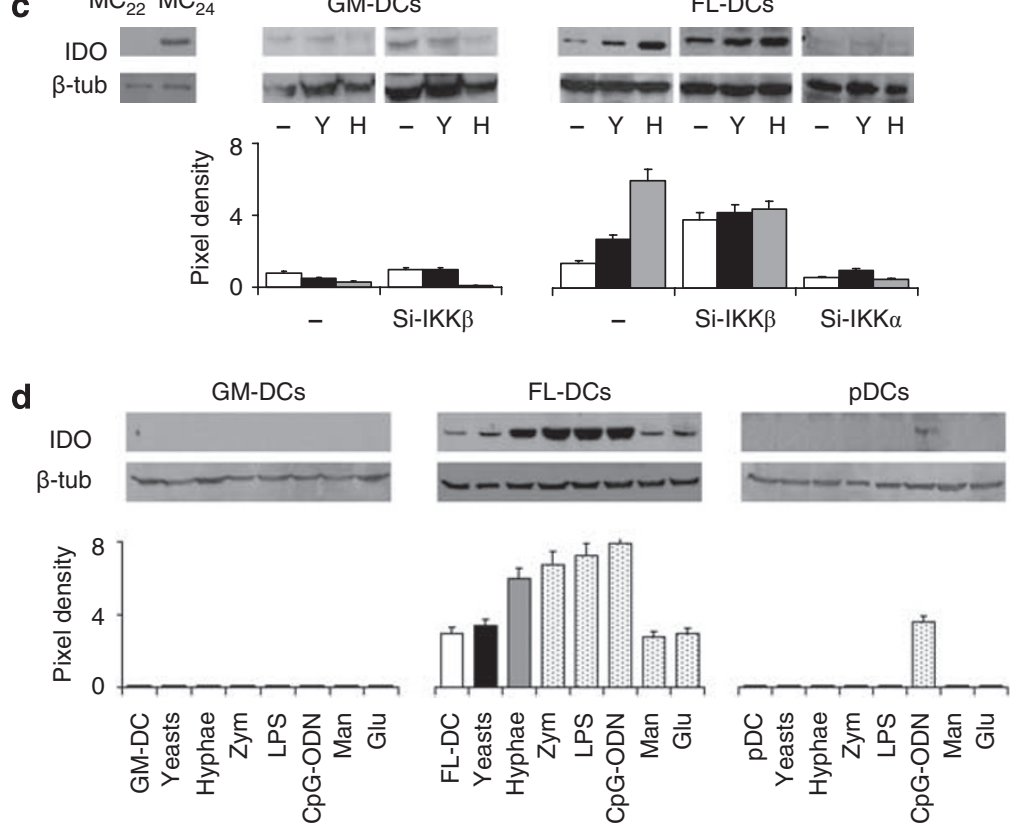

None

Yeasts

Hyphae

Figure 2 Activation of distinct nuclear factor-kappaB (NF-kB) pathways and 2,3 indoleamine dioxygenase (IDO) in dendritic cell (DC) subsets by Candida albicans. (a) Levels of IkappaB kinase-beta (IKK $\beta$ ) or IkapaB kinase-alpha (IKK $\alpha$ ) phosphorylation at 90 min of stimulation of bone marrow-derived GM-DCs or FL-DCs, from C57BL/6, myeloid differentiation factor-88 (MyD88), or Toll/IL-1 receptor domain-containing adaptor inducing IFN-beta (TRIF)-deficient mice, with live unopsonized Candida yeasts $(\mathrm{Y})$ or hyphae $(\mathrm{H})$. Data are presented as immunoblots of cell lysates with antiphospho-IKK $\alpha$ (Ser180)/IKK $\beta$ (Ser181) rabbit antibodies and fold increases (pixel density) in the phosphorylated to total protein ratios. (b) Canonical NF- $\kappa B$ activation in GM-DCs or FL-DCs at $180 \mathrm{~min}$ after fungal exposure by electrophoretic mobility shift assay (EMSA). For band specificity, the nuclear extracts were incubated with the antibodies to p65 and p50 before the probe was added. (c) IDO protein expression in DC subsets after $18 \mathrm{~h}$ of stimulation with yeasts or hyphae on silencing IKK $\beta$ or IKK $\alpha$ with small interference RNA (siRNA). Expression of IKK $\alpha$ and IKK $\beta$ transcripts was

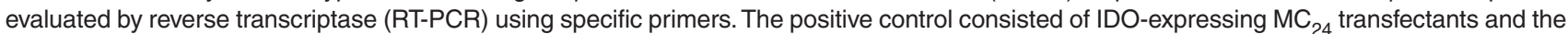
negative control consisted of mock-transfected $\mathrm{MC}_{22}$ cells. The pixel density of IDO band was normalized against $\beta$-tubulin ( $\beta$-tub). (d) IDO protein expression in DC subsets after 18h stimulation with live yeasts or hyphae, Zymosan (Zym), LPS, Glucan (Glu) or Mannan (Man) (10 ng/ml), or CpG oligodeoxynucleotide (CpG-ODN) $(2 \mu \mathrm{M})$. pDCs, plasmacytoid PDCA-1+ DCs fractioned from spleens. The values are representative of three experiments.

was dependent on p38, but not on ERK and on TRIF more than MyD88 (Figure 3b). STAT3 inhibition with Tyrphostin AG490 (AG490) (Figure 3d) and similarly with cucurbitacin I (JSI-124), a pharmacological inhibitor of the Jak2-STAT3 pathway (data not shown), enhanced the activation of the canonical pathway in both DC subsets (Figure 3c) but impaired the activation of the non-canonical pathway in FL-DCs (Figure 3d). STAT3 inhibition also greatly reduced IDO protein expression in FL-DCs, in a manner similar to p38 MAPK inhibition (Figure 3e). Together, these data indicate that STAT3 phosphorylation in response to hyphae is a proximal signaling for the activation of non-canonical NF- $\kappa B$ and IDO in FL-DCs. 


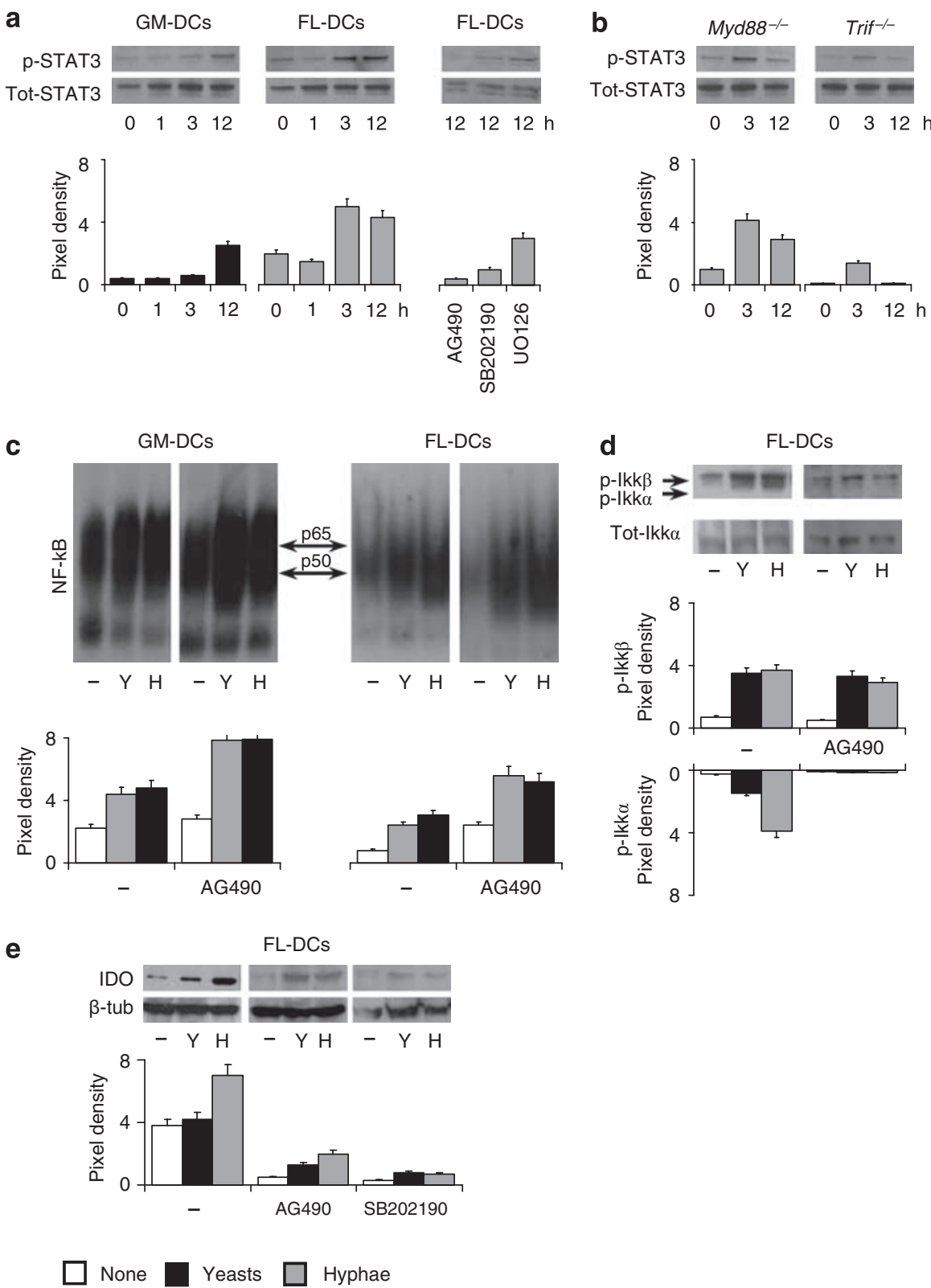

Figure 3 Activation of signal transducer and activator of transcription 3 (STAT3) in dendritic cell (DC) subsets by Candida albicans. (a) Phosphorylation of STAT3 in GM-DCs or FL-DCs, pulsed with yeasts or hyphae, (b) or in hypha-pulsed FL-DCs from myeloid differentiation factor-88 (MyD88)- or Toll/ IL-1 receptor domain-containing adaptor inducing IFN-beta (TRIF)-deficient mice. In panel a, STAT3 phosphorylation in hyphae-pulsed FL-DCs was assessed with and without the presence of inhibitors of STAT3 (Tyrphostin AG490 (AG490), $25 \mu \mathrm{M})$, p38 (SB202190, $5 \mu \mathrm{M})$, or ERK (UO126, 15 $\mu$ M). Blots of cell lysates were incubated with rabbit polyclonal Abs recognizing the phospho-STAT3 (Tyr705) followed by horseradish peroxidase-conjugated anti-rabbit IgG. Canonical nuclear factor-kappaB (NF-kB) activation by (c) electrophoretic mobility shift assay (EMSA) and (d) IkappaB kinase-beta $($ IKK $\beta$ ) or IkappaB kinase-alpha (IKK $\alpha$ ) phosphorylation at 90 min after stimulation in the presence of the STAT3 inhibitor AG490. (e) Immunoblots of IDO in FL-DCs at 18h after stimulation in the presence of AG490 or SB202190. $\beta$-tub, $\beta$-tubulin.

\section{The intracellular signaling pathways affect cytokine} production, maturation, and Notch ligand expression

Although a comprehensive analysis of cytokine/chemokine production by DC subsets performed with the Bio-Plex (BioRad Laboratories, Life Science Group, Segrate, Italy) cytokine assays is reported in Supplementary Figure S2, in this study, we addressed the relative contribution of each signaling pathway on the levels of IL-12p70, IL-23, IL-10, and IL-4 production, owing to the fact that the relative contribution of these cytokines to antifungal Th polarization is well described. ${ }^{1,2,5}$ In addition, we also assessed type I interferon (IFN) and IL-27 production, given the role of type I IFN on IDO induction ${ }^{25}$ and the anti-inflammatory property of IL-27. ${ }^{26}$ Cytokines were assessed both in terms of mRNA expression and actual cytokine production by either type of DCs in response to the different stimuli in the presence of inhibitors of ERK1/2, p38, JNK1/2, PI3K-Akt, STAT3, and canonical (by si-IKK $\beta$ ) or non-canonical (by si-IKK $\alpha$ ) NF$\kappa B$. Confirming earlier data, ${ }^{2}$ levels of p35 and p19 mRNA were 


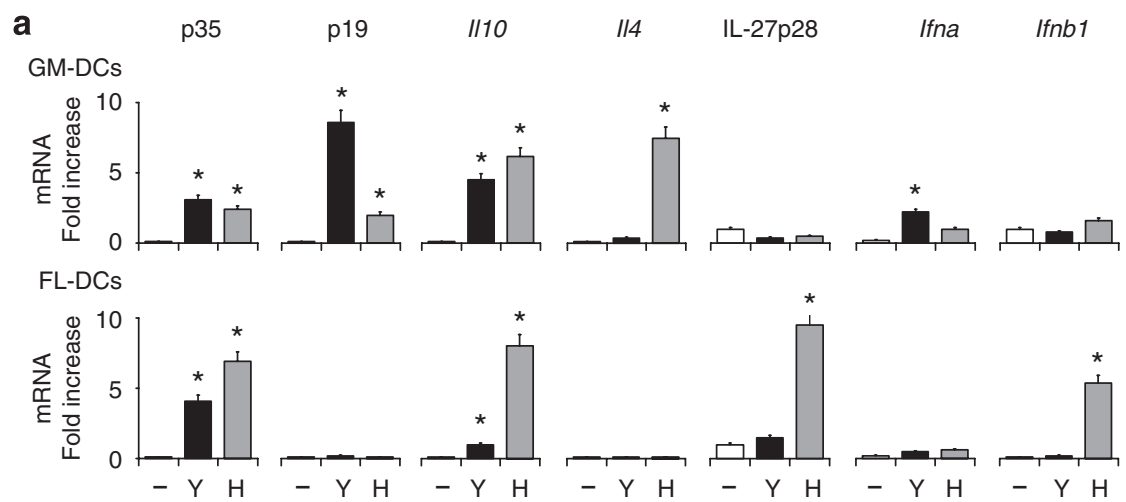

\section{b}

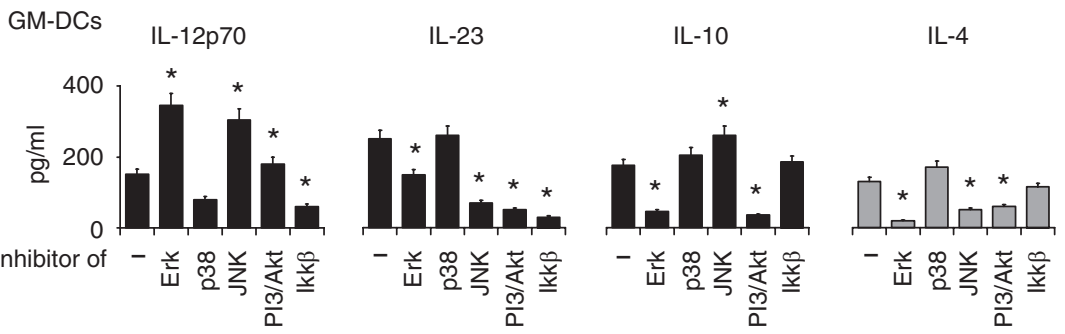

FL-DCs

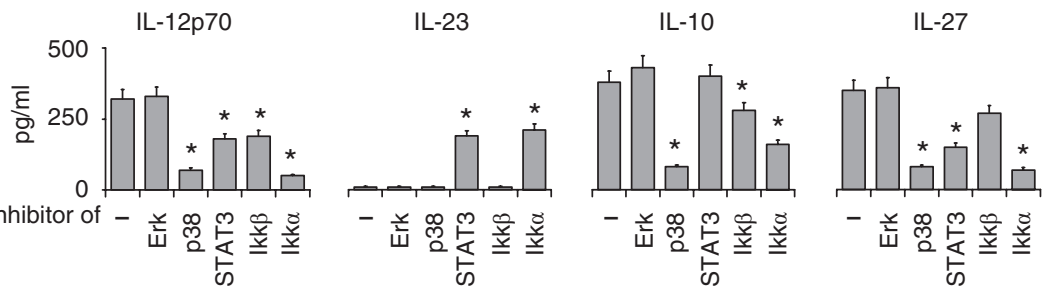

C

GM-DCs

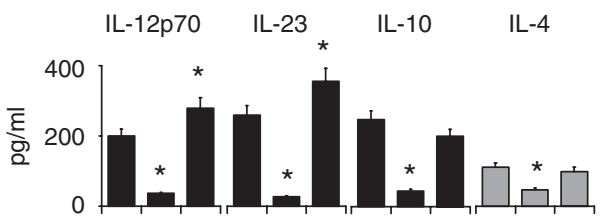

FL-DCs

IL-12p70 IL-10

IFN- $\beta$

IL-27 d

GM-DCs

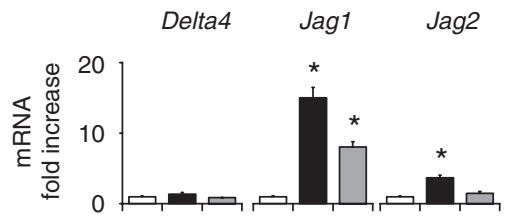

FL-DCs
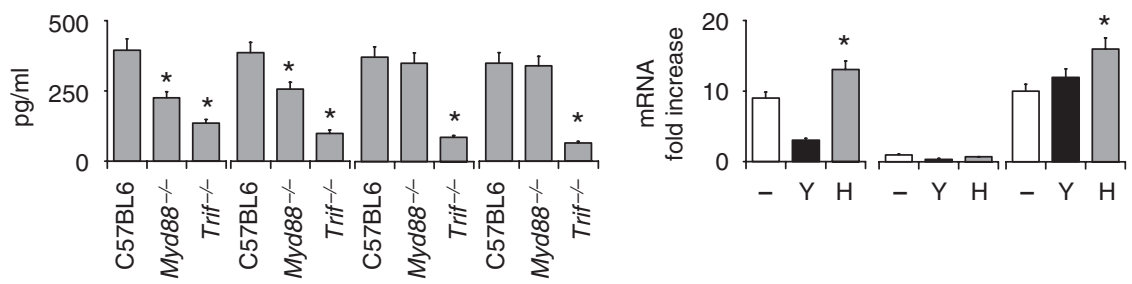

None

Yeasts

Hyphae

Figure 4 The intracellular signaling pathways affect cytokine production and maturation of dendritic cell (DC) subsets exposed to Candida. Cytokines were assessed in terms of mRNA expression by (a) reverse transcriptase PCR (RT-PCR) or (b, $\mathbf{c})$ cytokine production by ELISA by each DC subset unstimulated $(-)$ or stimulated with yeasts or hyphae (black and gray histograms, respectively) in the presence of inhibitors of extracellular signalregulated kinases (ERK1/2), (U0126), p38 (SB202190), Jun N-terminal kinases 1 and 2 (JNK1/2) (10 $\mu \mathrm{m}$, SP600125), phosphoinositide 3-kinase (PI3K)-Akt ( $5 \mu \mathrm{M}$, Wortmannin), signal transducer and activator of transcription 3 (STAT3) (AG490), and canonical (si-lkappaB kinase-beta (IKK $\beta)$ ) or non-canonical (si-IkappaB kinase-alpha (IKKa)) nuclear factor-kappaB (NF-kB). (d) Expression of Notch ligands in unstimulated (-) or Candidastimulated DCs by RT-PCR. Jag1 and Jag2, Jagged1 and Jagged2. ${ }^{*} P<0.05$, pulsed vs. unpulsed ( - ) DCs; pulsed DCs with and without inhibitors, and C57BL/6 DCs vs. Myd88 ${ }^{-/-}$or Trif ${ }^{/-}$DCs. The values are representative of five experiments. 

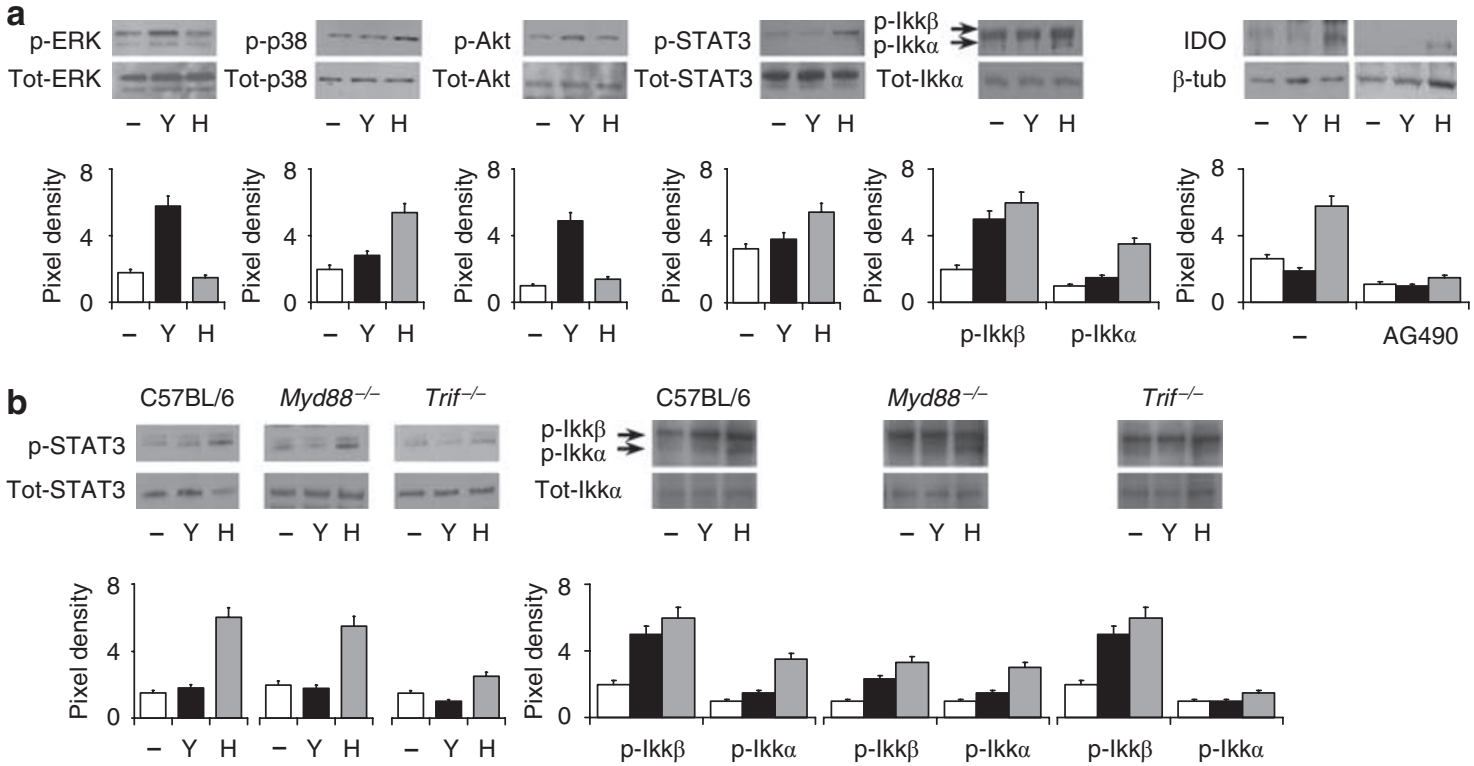

$\square$ None $\square$ Yeasts

Hyphae

Figure 5 Distinct intracellular signaling pathways are activated by yeasts and hyphae in PP-DCs. (a) Peyer's patch (PP) DCs from naive mice were exposed in vitro to live unopsonized Candida yeasts (black histograms) or hyphae (gray histograms) before the assessment of extracellular signalregulated kinases (ERK1/2), p38 mitogen-activated protein kinase (MAPK), Akt, signal transducer and activator of transcription 3 (STAT3), IkappaB kinase-beta (IKK $\beta$ ) or IkappaB kinase-alpha (IKK $\alpha$ ) phosphorylation (30 min later), and IDO protein expression (with and without Tyrphostin AG490 (AG490), after 18 h stimulation). (b) PP-DCs were purified from naive or C57BL/6-, myeloid differentiation factor-88 (MyD88)-, or Toll/IL-1 receptor domain-containing adaptor inducing IFN-beta (TRIF)-deficient mice (a day after the intragastric infection with $10^{8} \mathrm{Vir}^{-13}$ or Vir-3 cells) and immediately assessed for STAT3, IKK $\beta$, or IKK $\alpha$ phosphorylation. Data are presented as immunoblots of cell lysates with phosphorylation-specific antibodies and fold increases (pixel density) in the phosphorylated to total protein ratios or to $\beta$-tubulin ( $\beta$-tub). Representative of three experiments.

higher in GM-DCs upon yeast rather than hyphae stimulation, and levels of $I l 10$ were comparable, whereas those of $I l 4$ were higher with hyphae rather than with yeast stimulation. By contrast, levels of Il10, IL27p28, and Ifn- $\beta$ were higher in FL-DCs stimulated with hyphae than with yeasts, whereas those of $\mathrm{p} 35$ were comparable and no p19 mRNA could be detected (Figure 4a). Results with the inhibitors showed that cytokine production by GM-DCs was largely p38-independent and mostly, though variably, regulated by ERK-JNK-PI3K and canonical NF- $\mathrm{\kappa B}$. Although both were dependent on canonical NF- $\kappa B$, IL-12p70 was inhibited by ERK and JNK, but IL-23 was promoted by ERK, JNK, and PI3K. By contrast, IL-10 was dependent on ERK and PI3K and was negatively regulated by JNK, IL-4 was ERK-, JNK-, and PI3K-dependent, and both were largely independent from canonical NF- $\mathrm{KB}$. In FL-DCs, the production of cytokines was largely independent from ERK and was dependent on p38 and non-canonical NF- $\kappa B$ (IL-12p70, IL-10, and IL-27), as well as on STAT3 (IL-12p70 and IL-27). Interestingly, the inhibition of STAT3 and non-canonical NF- $\kappa$ B promoted IL- 23 production in these cells (Figure 4b). Experiments with $M y d 88^{-/-}$DC or Trif $^{-1-}$ DCs confirmed that cytokine production by GM-DCs was MyD88-dependent, whereas that of FL-DCs was mostly (IL-12p70) or totally (IL-10, IFN- $\beta$, and IL-27) TRIF-dependent (Figure 4c). These data indicate that cytokine production in GM-DCs occurs mainly through MyD88 and implicates ERK-JNK-PI3K-Akt and canonical NF-אB signaling. By contrast, the TRIF pathway essentially regulates cytokine production by FL-DCs and implicates p38, STAT3, and non-canonical NF$\kappa \mathrm{B}$ signaling.

In terms of maturation and costimulatory molecule expression, GM-DCs expressed CD80 more than CD86 in an ERKdependent manner, whereas FL-DCs expressed CD86 and the maturation-marker CD83 in a p38-dependent manner. As costimulatory molecules, known to be involved in Treg activation by DCs, such as inducible costimulator ligand (ICOSL), glucocorticoid-induced tumor necrosis factor receptor ligand (GITRL) and programmed cell death-1 ligand (PD-L1), were not induced in either of the DC subsets (data not shown), and given the important role of the Notch ligands, Delta 4 and Jagged 1 and 2 , in setting the outcome of immune reactivity, ${ }^{27}$ we assessed the expression of Delta 4, Jagged 1 and 2 on DC subsets. Both Delta 4 and Jagged 2 expressions were upregulated in FLDCs, whereas the expression of Jagged 1 was upregulated in GM-DCs (Figure 4d). As the expression of Delta 4 in DCs is associated with the activation of IL-10-producing Th1 cells and Jagged 1 and 2 expressions are variably involved in the induction of Th2/Treg cells, ${ }^{27}$ these data would predict that either type of the DC subsets is competent for the activation of distinct adaptive Th responses in vivo.

\section{Activation of distinct intracellular programs in Peyer's patch DCs by Candida}

To define the in vivo relevance of the above findings, we assessed Peyer's patches (PP)-DCs for signal transduction pathways 
a

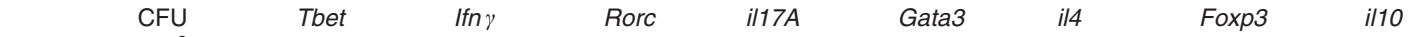

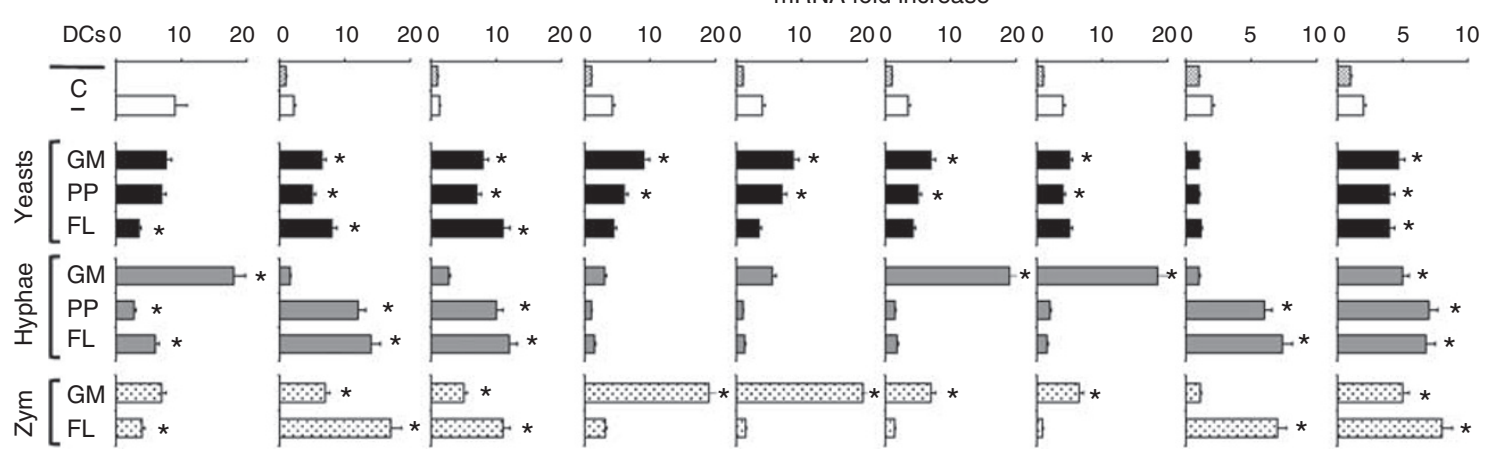

b
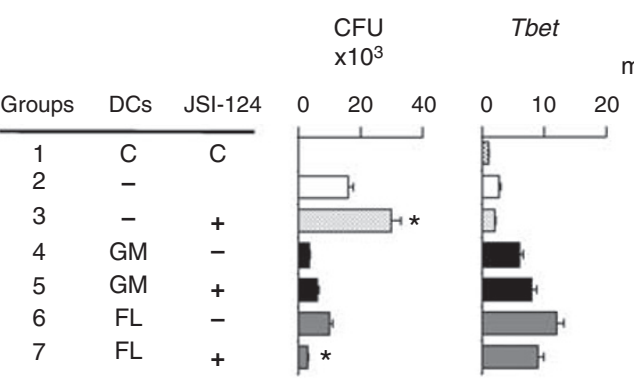

Rorc

Foxp3

mRNA fold increase

$\begin{array}{llllll}0 & 10 & 20 & 0 & 5 & 10\end{array}$

c
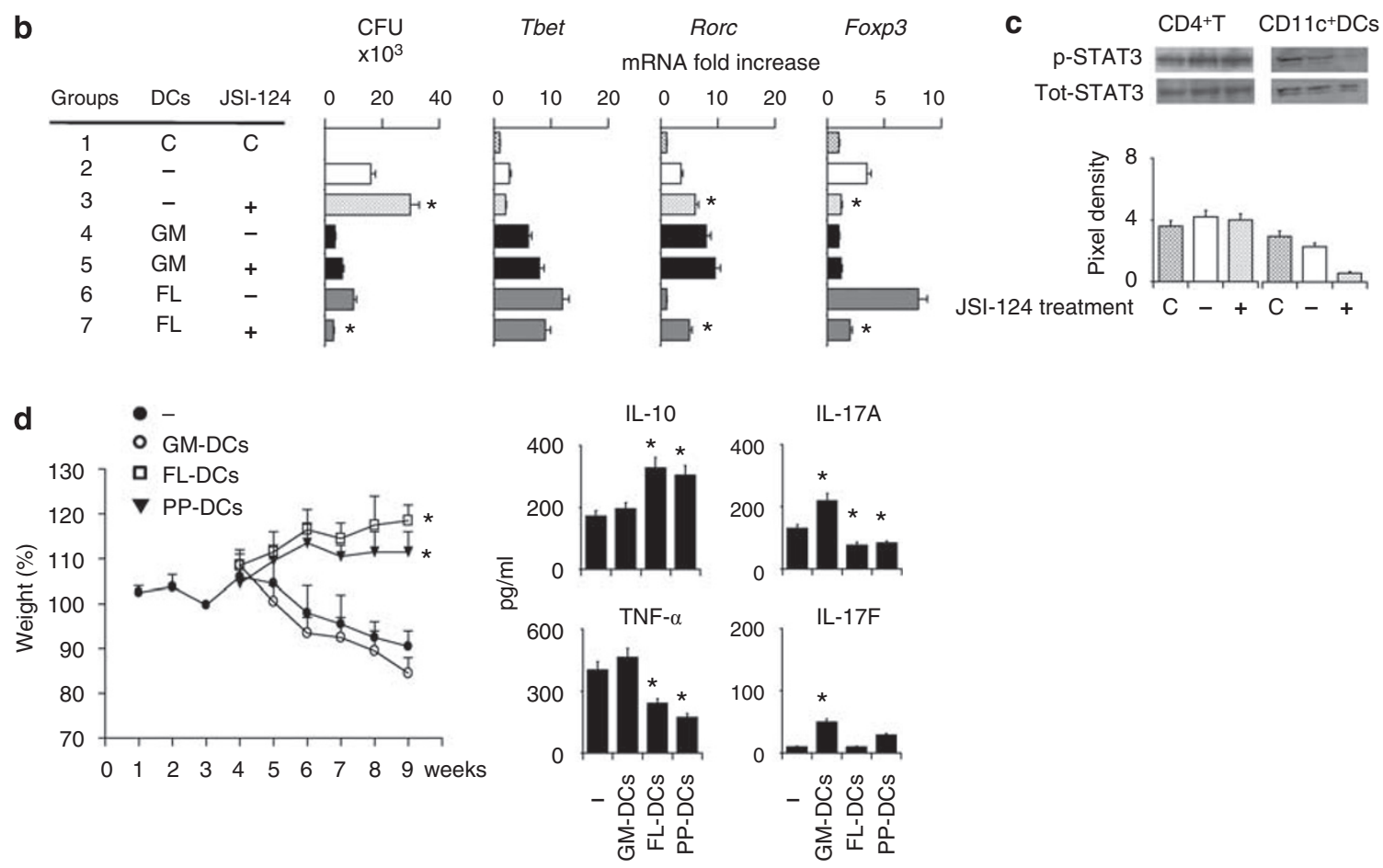

Figure 6 Peyer's patch (PP) DCs activate distinct antifungal Th cell responses in vivo and attenuate inflammation in the gut. (a) C57BL/6 mice received Candida- or Zymosan (Zym)-pulsed DCs twice, a week apart, before the intragastric infection with the fungus. Fungal growth colony-forming units (CFU) in the stomach, Th lineage-specific transcription factors, and cytokine expression in CD4 ${ }^{+} \mathrm{T}$ cells from mesenteric lymph nodes by reverse transcriptase (RT-PCR) were assessed a week after the infection. ${ }^{*} P<0.05$, DC-treated vs. untreated $(-)$ mice. $\mathrm{C}$, control naive mice. The values are representative of three experiments. (b) C57BL/6 mice intragastrically infected with the fungus were treated with $1 \mathrm{mg} / \mathrm{kg}$ cucurbitacin I (JSI-124) for 3 days post infection and assessed for fungal growth and Th-specific transcription factors as in panel a (groups 2 and 3). Groups 4-7 refer to mice treated with GM-DCs or FL-DCs in vitro pulsed with yeasts or hyphae, respectively, in the presence of JSI-124 $(10 \mu \mathrm{M})$. ${ }^{*} P<0.05$, treated vs. untreated (-) mice and FL-DCs with and without JSI-124. (c), signal transducer and activator of transcription 3 (STAT3) phosphorylation in purified CD4 ${ }^{+} \mathrm{T}$ cells or CD11+DCs from the mesenteric lymph nodes of mice treated (+) as in panel a. C, control naive mice. (d) Yeast-pulsed GM-DCs and hyphae-pulsed FL-DCs or PP-DCs were injected into severe combined immune deficiency (SCID) mice $3 \mathrm{~h}$ before administration of colitogenic CD4+ ${ }^{+}$D25- $\mathrm{T}$ cells from BALB/c mice. Mouse weight was registered twice per week, and mice were killed around day 60 . Data are mean $\pm s$.e. of mice in each treatment group $(n=6)$ in one representative experiment out of two. Cytokines were determined in colon homogenates 9 weeks later. ${ }^{*} P<0.05$, FL-DC- or PP-DC treated vs. untreated $(-, \bullet)$ mice.

activated in response to yeasts and hyphae, in vitro and in vivo. Similar to GM-DCs, ERK and Akt phosphorylation and canonical NF- $\kappa \mathrm{B}$ activation occurred in PP-DCs pulsed in vitro with yeasts, whereas, similar to FL-DCs, p38 phosphorylation, activation of STAT3, non-canonical IKK $\alpha$, and induction of STAT3-dependent IDO expression, occurred in response to hyphae (Figure 5a). Results from infected mice confirmed the in vitro findings and showed that IKK $\beta$ activation was, albeit partially, MyD88dependent, whereas STAT3 and IKK $\alpha$ activation in response to hyphae was TRIF-dependent (Figure 5b). As IDO activation in PP-DCs in response to hyphae in vivo also occurred in a TRIFdependent manner, ${ }^{2}$ these data indicate that the STAT3-noncanonical NF- $\kappa \mathrm{B}-\mathrm{IDO}$ pathway is activated in PP-DCs by hyphae and in the ERK-Akt-canonical NF-kB pathway by yeasts. 


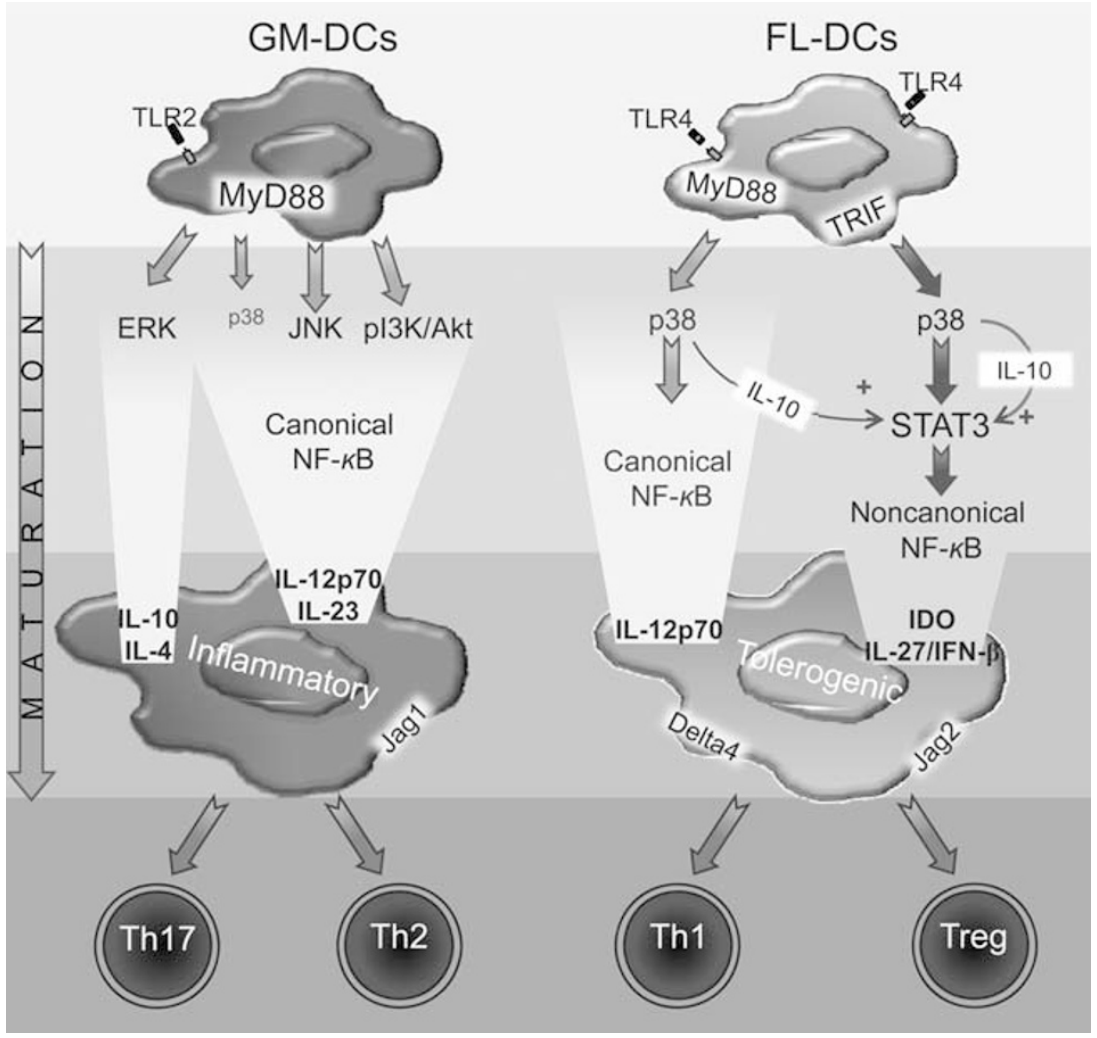

Figure 7 Scheme of putative signaling pathways accounting for specialization and flexibility of DCs presenting Candida albicans. The figure shows that the balance between distinct signaling pathways in DCs translates Toll-like receptor (TLR) dependent recognition of Candida morphotypes into distinct adaptive immune responses. IDO, 2,3 indoleamine dioxygenase. See text for details.

\section{PP-DCs activate distinct antifungal Th/Treg cell responses in vivo}

To assess whether the differential molecular profiles elicited in PP-DCs translate into distinct adaptive Th responses in vivo, yeast- or hyphae-pulsed PP-DCs were comparatively analyzed with similarly pulsed GM-DCs or FL-DCs for their ability to activate immune protection in mice with candidiasis. Figure $\mathbf{6 a}$ shows that PP-DCs or GM-DCs pulsed with yeasts promoted the activation of $\operatorname{Rorc}^{+} 17$-producing CD4 ${ }^{+} \mathrm{T}$ cells and Gata3 ${ }^{+}$ IL-4/IL-10-producing CD ${ }^{+} \mathrm{T}$ cells with minimal activation of tbet ${ }^{+}$IFN- $\gamma$-producing cells, and this resulted in a limited control of fungal growth.

Similar to FL-DCs, hyphae-pulsed PP-DCs promoted the activation of Th1/Treg cells, indicating the activation of a protective tolerogenic response to the fungus that accounted for the control of fungal growth and long-term survival (Figure 6a). These data indicate that distinct signal transduction pathways mediate the functional activity of DCs in vivo. This was corroborated by the findings with selected fungal PAMPs. Zymosan, known to induce both $\mathrm{Th}_{17}{ }^{28}$ and Tregs, ${ }^{29}$ activated GM-DCs for Th17 priming and FL-DCs for Th1/Treg priming (Figure 6a), whereas mannan-pulsed GM-DCs, mimicking hyphae-pulsed GM-DCs, promoted the activation of Gata3 ${ }^{+}$Th2 cells associated with extensive fungal growth (data not shown). These data suggest that inflammatory DCs activate and regulatory DCs attenuate inflammatory responses to the fungus and are in line with the findings that inflammation is attenuated in MyD88-deficient mice and promoted in TRIF-deficient mice, and that tolerogenic $\mathrm{IDO}^{+} \mathrm{DC}$ reinstate tolerance in the otherwise intolerant mice. $^{2,11}$

As defective STAT3 has been linked to candidiasis, ${ }^{30,31}$ we blocked STAT3 activation in infection or in Candida-pulsed DCs before the adoptive transfer in vivo by treatment with JSI124, a pharmacological inhibitor of the Jak2-STAT3 pathway. ${ }^{32}$ Figure $\mathbf{6 b}$ shows that treatment with JSI-124 greatly increased the fungal growth in the stomach and activated Rorc ${ }^{+}$ but not $\mathrm{Tbet}^{+}$or Foxp3 ${ }^{+} \mathrm{CD}^{+} \mathrm{T}$ cells in mesenteric lymph nodes (MLN). On assessing which cell types ( $\mathrm{T}$ vs. myeloid cells) were affected by JSI-124 treatment in vivo, STAT3 activation was impaired in CD $11 \mathrm{c}^{+}$but not in CD $4^{+} \mathrm{T}$ cells (Figure 6c), a finding in line with the notion that, at variance with $\mathrm{T}$ cells, ${ }^{30,31}$ STAT3 deficiency in myeloid DCs promotes inflammatory diseases. ${ }^{33}$ As predicted by the increased IL-23 production (Figure 4b), blocking STAT3 abrogated the capacity of FL-DCs to activate tolerogenic responses to the fungus and resulted in Th17 cell activation (Figure $\mathbf{6 b}$ ). Together, these data suggest that STAT3 activation in myeloid cells is required for the occurrence of tolerogenic responses to the fungus ultimately affecting the local Th17/Treg balance.

\section{Candida attenuates the inflammatory response in the gut through PP-DCs}

The finding that STAT3 deficiency in myeloid cells causes Crohn's disease-like pathogenesis and lethality, ${ }^{33,34}$ and IDO 
induction attenuates colitis ${ }^{35}$ and mediates probiotic functionality, ${ }^{36}$ led us to hypothesize whether immune regulation by Candida through PP-DCs could affect inflammation in experimental colitis. On adoptive transfer in severe combined immune deficiency (SCID) mice with $\mathrm{CD} 4{ }^{+} \mathrm{CD} 25^{-} \mathrm{T}$ cell-induced colitis, Candida-pulsed PP-DCs and FL-DCs, but not GM-DCs, prevented weight loss, and restored the inflammatory/antiinflammatory cytokine balance (Figure 6c). Thus, tolerogenic PP-DCs may serve the dual role of inducing protective tolerance to the fungus-likely favoring commensalisms - as well as attenuating the local inflammatory response in the gut.

\section{DISCUSSION}

C-type lectin receptors, Dectin- ${ }^{37-39}$ and dendritic cell-specific intercellular adhesion molecule 3-grabbing nonintegrin (DCSIGN) ${ }^{40}$ are known to regulate IL-10 and IL-12 production by DCs in response to Candida or fungal PAMPs, and to contribute to antifungal immunity and immune homeostasis. ${ }^{29,41}$ However, a comprehensive analysis of the relative contribution of intracellular signaling pathways and/or distinct DC subsets in sensing Candida morphotypes has not been reported. In this study, we show that the balance between distinct signaling pathways in DCs translates the TLR-dependent recognition of Candida morphotypes into distinct adaptive immune responses in vivo through the antagonistic MyD88 and TRIF pathways (Figure 7). The ERK-c-fos conditioned GM-DCs both for Th2 and, along with JNK and PI3K-Akt, for Th17 priming, whereas the p38 pathway conditioned FL-DCs for Th1/Treg priming. The TLR2-MyD88 pathway essentially mediated the activation of inflammatory GM-DCs, whereas the TLR4-TRIF pathway was critical for the activation of tolerogenic FL-DCs. As the hyperphosphorylation of $c$-Jun and $c$-fos transcription factors is known to mediate Th2 activation by DCs, ${ }^{13}$ the fact that hyphae, more than yeasts, activated reactive oxygen species (ROS) in DCs, ${ }^{15}$ and that persistent JNK activation is mediated by ROS, may offer a plausible explanation for the higher Th2-promoting activity of hypha-pulsed DCs.

At variance with hyphae, yeasts also activated GM-DCs with Th17-polarizing capacity, as predicted by the production of IL-23 and IL-1 $\beta$ (Supplementary Figure S2), both cytokines being required for Th17 activation in response to fungi. ${ }^{42}$ This occurred through the TLR2/MyD88-dependent activation of the PI3K-Akt pathway, known to suppress IL-12 and to promote IL-10 on TLR2 activation of DCs. ${ }^{43}$ The finding that this signaling pathway was activated, albeit late, in response to hyphae suggests the possible contribution of hyphae to IL-23 production by DCs, as suggested. ${ }^{44}$ Glucan-pulsed GM-DCs also induced Th17 cell activation in vivo (data not shown), a finding confirming the contribution of dectin-1 agonists to Th17 activation ${ }^{45}$ and autoimmunity. ${ }^{41}$

In contrast to GM-DCs, a sustained p38 favored a Th1/Treg outcome in FL-DCs stimulated with hyphae, confirming the pivotal role of the $\mathrm{p} 38$ pathway in IL-12p70 production through TLR4 and DC maturation. ${ }^{46}$ Thus, rather than eluding TLR4 signaling, as suggested with mononuclear cells, ${ }^{7,8}$ Candida hyphae are particularly adept at activating the TLR4-TRIF pathway in
DCs, a finding supported by the notion of a differential requirement for TRIF-MyD88 signaling pathways in the activation of DCs vs. macrophages. ${ }^{16}$ As a matter of fact, the production of cytokines associated with tolerogenic DCs, namely IL-10, IFN- $\beta$, and IL-27, was strictly p38/TRIF-dependent. Similar to hyphae, zymosan activated the tolerogenic program of FL-DCs. Thus, the capacity of zymosan to activate inflammatory and tolerogenic DCs may offer a plausible explanation for the apparently conflicting role of zymosan in immune activation, being associated with both Th1 $7^{28,47}$ and $\mathrm{Treg}^{29}$ cell activation in vivo.

One important and novel observation of this study is the contribution of STAT3 to the plasticity of DC subsets in response to the fungus. STAT3 was activated in FL-DCs through TRIF and p38, mediated downstream by non-canonical NF- $\mathrm{KB} / \mathrm{IDO}$ activation, and likely occurred through the IL-10/IL-10R autocrine loop, being inhibited on IL-10 blockade (data not shown) and less through the IL-6-gp-130-STAT3 pathway, as IL-6 production in FL-DCs was not obviously different between hyphae and yeasts (Supplementary Figure S2). It is of interest that glycogen synthase kinase-3, known to favor the nucleocytoplasmic shuttling of STAT $3,{ }^{48}$ was also activated more in FL-DCs than in GM-DCs.

However, the late STAT3 expression in GM-DCs suggests a possible two-step model of activation, in which the late activation of STAT3/IL-10 by proinflammatory stimuli may serve to limit the activation state of DCs and to contribute to the production of IL- 10 by Th 1 cells. The finding that STAT3 and the non-canonical NF- $\kappa B$ blockade both resulted in IL-23 production provides evidence of the contribution of this pathway to the interconvertibility between DC subsets, thus further adding to the flexibility and plasticity of DC responses in vivo. In this regard, the in vivo data obtained by selectively blocking STAT3 activation in DCs are in line with the findings of an increased susceptibility to infection in patients with defective STAT3 activation. ${ }^{30,31}$ However, consistent with the overt autoimmunity that develops in myeloid-specific STAT3-deficient mice, ${ }^{33}$ defective tolerance seems to be a mechanism by which candidiasis is promoted under conditions of STAT3 deficiency in myeloid cells. Although the deficit in Th17 has been attributed to susceptibility to candidiasis in patients with autosomal-dominant hyper-IgE syndrome and STAT3 deficiency, ${ }^{30,31}$ whether DC development is also impaired in these patients remains to be determined.

Finally, the finding that PP-DCs, similar to FL-DCs, activate the tolerogenic program in response to Candida hyphae and that both DC types ameliorate colitis points to the fungus as a commensal with local immunoregulatory functions through DCs. We have evidence that Candida-induced Tregs also ameliorates colitis through reverse signaling on PP-DCs (manuscript in preparation). This would suggest that, similar to probiotics ${ }^{36}$ but different from Citrobacter rodentium, ${ }^{49}$ attenuation of colitis is mediated by IDO + DCs. The fact that human DC subsets, similar to the murine counterparts, also activate discriminative T-cell responses to the fungus (Supplementary Figure S3) point to C. albicans as a commensal capable of balancing inflammation and tolerance at mucosal sites through the exploitation of intracellular antagonistic pathways in DCs. 
Ultimately, this may indicate a high degree of co-evolution of the mammalian host and C. albicans and the resultant symbiosis between the two.

\section{METHODS}

Mice. Female C57BL/6, BALB/c, and SCID mice, 8-10 weeks old, were purchased from Charles River (Calco, Italy). Homozygous $T l r 2^{-/-}$, Tlr4 ${ }^{-1-}$, Myd88 $8^{-1-}$, and Trif ${ }^{-1-}$ mice on a C57BL/6 background were bred under specific pathogen-free conditions at the Animal Facility of Perugia University, Perugia, Italy. Experiments were performed according to the Italian Approved Animal Welfare Assurance A-3143-01.

Fungal strains and infections. Isogenic strains of $C$. albicans, obtained by mutagenesis in vitro and capable ( $\left.\mathrm{Vir}^{-} 13\right)$ or not $\left(\mathrm{Vir}^{-} 3\right)$ of yeastto-hypha transition, as assessed by the germ-tube formation in vitro, were used. ${ }^{2,5}$ For hyphae, cells were allowed to germinate by culture at $37^{\circ} \mathrm{C}$, in $5 \% \mathrm{CO}_{2}$, for $2 \mathrm{~h}$ in RPMI 1640 medium (by that time, $>98 \%$ of cells had germinated). For yeasts, cells were harvested at the end of the exponential phase of growth. For infection, mice received $10^{8} \mathrm{Vir}^{-} 13$ cells intragastrically and were treated with JSI-124 (cucurbitacin I, from Tocris Bioscience, Bristol, UK) or vehicle control (DMSO) for 3 days, at $1 \mathrm{mg} / \mathrm{kg}$, a dose earlier shown to induce effective antitumor effects in. ${ }^{32}$

Cell preparation and culture. GM-DCs or FL-DCs were obtained by culturing bone marrow cells with $150 \mathrm{U} / \mathrm{ml}$ mouse rGM-CSF (Sigma, St Louis, MO) and 75 U/ml rIL-4 (R\&D Systems, Milan, Italy) in Iscove medium supplemented with glutamine, penicillin and streptomycin, and $10 \%$ heat-inactivated fetal calf serum for 7 days or $200 \mathrm{ng} / \mathrm{ml}$ FLT3-L (R\&D Systems, Milan, Italy) for 9 days. ${ }^{11}$ For the positive selection of $\mathrm{mPDCA}-1^{+}$pDCs, splenic CD $11 \mathrm{c}^{+}$cells were fractioned using mPDCA-1 MicroBeads (Miltenyi Biotec, Bergisch Gladbach, Germany). More than $95 \%$ of the mPDCA- $1^{+}$cells were stained by the $120 \mathrm{G} 8$ marker. ${ }^{11}$ PP-DCs were isolated as described. ${ }^{12}$ Inhibitors of ERK1/2 (15 $\mu \mathrm{M}, \mathrm{U} 0126), \mathrm{p} 38(5 \mu \mathrm{M}, \mathrm{SB} 202190), \mathrm{JNK} 1 / 2(10 \mu \mathrm{m}$, SP600125), PI3K-Akt (5 $\mu \mathrm{M}$, Wortmannin; Sigma), and STAT3 $(25 \mu \mathrm{M}$, AG490) were purchased from Calbiochem (San Diego, CA) and dissolved at $1,000 \times$ the final concentration in DMSO (Sigma). DCs were pre-exposed to the inhibitors for $120 \mathrm{~min}$ at $37^{\circ} \mathrm{C}$ before stimulation. Control cells were treated with an identical amount of DMSO. Stimuli included Zymosan $(10 \mathrm{ng} / \mathrm{ml})$ from Saccharomyces cerevisiae (Sigma), ultra-pure lipopolysaccharide $(10 \mathrm{ng} / \mathrm{ml})$ from Salmonella minnesota Re 595 (Labogen S.r.l., Rho, Milan, Italy), cytosine-phosphorothioateguanine oligodeoxynucleotide $(2 \mu \mathrm{M}$, CpG-ODN 1826, Invitrogen Life Technologies, Carlsbad, CA), mannan from S. cerevisiae or barley $\beta$-glucan (both at $10 \mathrm{ng} / \mathrm{ml}$, Sigma), live yeasts, or hyphae ${ }^{11}$ in Iscove medium supplemented as above without serum.

Adoptive transfer of DCs, fungal challenge, and assessment of protection. After $2 \mathrm{~h}$ of pulsing, DCs $\left(5 \times 10^{5}\right)$ were administered intraperitoneally twice in $20 \mu \mathrm{l}$ of phosphate-buffered saline, at weekly intervals, before the infection. A week later, mice were assessed for fungal growth and Th/Treg cell activation by reverse transcriptase PCR (RT-PCR) on purified (Miltenyi Biotec) $\mathrm{CD}^{+}(>98 \%$ ) T cells from draining mesenteric lymph nodes.

Induction of colitis. CD4 ${ }^{+} \mathrm{CD} 25^{-} \mathrm{T}$ cells $\left(3 \times 10^{5}\right)$, purified from MLN of BALB/c mice (Miltenyi Biotec), were transferred intraperitoneally to SCID mice $3 \mathrm{~h}$ after the intraperitoneal injection of $3 \times 10^{5}$ Candida-pulsed GM-DCs, FL-DCs, or PP-DCs. The mice were then monitored once or twice a week for weight loss, loose stools, bloody diarrhea, and rectal prolapse. ${ }^{50}$ Colon homogenates were obtained from collagenase $(1 \mathrm{mg} /$ $\mathrm{ml}$, Clostridiopeptidase A, Sigma) and DNase ( $5 \mu \mathrm{g} / \mathrm{ml}$, Boehringher Mannheim, Mannheim, Germany)-treated colons.
Western blotting. Protein phosphorylation was assessed on cell lysates incubated with rabbit polyclonal Abs, recognizing the unphosphorylated form of ERK, p38, JNK, Akt, STAT3 or phosphop44/p42 ERK (Thr202/Tyr294), phospho-p38 (Thr180/tyr182), phospho-SAPK/ JNK (Thr 183/Tyr185), phospho-Akt (Ser 473), and phospho-STAT3 (Tyr705) followed by horseradish peroxidase-conjugated anti-mouse or anti-rabbit IgG, as per the manufacturer's instructions (Cell Signaling Technology, Danvers, MA). Blots were developed using the Enhanced Chemiluminescence detection kit (Amersham Pharmacia Biotech, Milan, Italy). Immunoblotting for IDO was performed with rabbit polyclonal IDO-specific antibody on DCs after $18 \mathrm{~h}$ of stimulation. ${ }^{2}$ The positive control consisted of IDO-expressing $\mathrm{MC}_{24}$ transfectants, and the negative control consisted of mock-transfected $\mathrm{MC}_{22}$ cells. Scanning densitometry was performed on a Scion Image apparatus (Scion Corporation, $\mathrm{MD}$ ). The pixel density of bands was normalized against total proteins or $\beta$-tubulin.

Canonical and non-canonical NF-кB. For electrophoretic mobility shift assays, the double-stranded probe containing an NF- $\kappa$ B consensus site 5agttgaggggactttcccaggc-3-was terminally labeled with $\mathrm{T} 4$ polynucleotide kinase 3'-phosphatase (PNK). The experimental reaction of the electrophoretic mobility shift assay experimental reaction contained $10 \mu \mathrm{g}$ of nuclear extracts, $2 \mu \mathrm{g}$ of non-specific competitor poly (dI-dC), and $200 \mathrm{ng}$ of single-stranded oligonucleotide. The binding reaction mixture was prepared with 50,000 c.p.m. ( $40 \mathrm{fmol}$ ) of radiolabeled probe for $20 \mathrm{~min}$ in 20 mM 4-(2-hydroxyethyl)-1-piperazineethanesulfonic acid (HEPES) (pH 7.6), $100 \mathrm{mM} \mathrm{NaCl}, 1 \mathrm{mM}$ dithiothreitol, $1 \mathrm{mM}$ phenylmethanesulphonylfluoride or phenylmethylsulphonyl fluoride (PMSF), $1 \times$ complete protease inhibitor mixture (Roche Molecular Biochemicals, Mannheim, Germany), and 5\% glycerol. Complexes were resolved on a $6 \%$ native polyacrylamide gel for $120 \mathrm{~min}$ at $170 \mathrm{~V}$ in Tris-borate-EDTA (TBE) $0.5 \times$. After electrophoresis, the gel was dried and processed for autoradiography. For band specificity, the nuclear extracts were incubated with the antibodies to p65 and p50 (Santa Cruz Biotechnology, Santa Cruz, CA) for $45 \mathrm{~min}$ at $4^{\circ} \mathrm{C}$ before the probe was added. Anti-phospho-IKK $\alpha(\operatorname{Ser} 180) /$ IKK $\beta$ (Ser181) rabbit Abs (Cell Signaling Technology) were used for the western blotting of phospho IKK $\alpha$ and IKK $\beta$.

siRNA synthesis and transfection. siRNAs were performed to target IKK $\alpha$ and IKK $\beta$ as described. ${ }^{19}$ The siRNA sequences specific for mouse $I K K \beta$ (alias $I k b k b$; sense, $5^{\prime}$-GGUGCAUUCAUUAUCUUA Att- ${ }^{\prime}$; antisense, $5^{\prime}$-UUAAGAUAAUGAAUGCACCtg- $3^{\prime}$ ) or $I K K \alpha$ (alias Chuk; sense, $5^{\prime}$-GGAAUAAAUACAGGUUCUCtt- $3^{\prime}$; antisense, 5'-GAGAACCUGUAUUUAUUCCtg-3') were selected, synthesized, and annealed by the manufacturer, and were used in combination with a negative control siRNA (Ambion, Austin, TX). DOTAP (Sigma) $(1,2$ dioleolyl-3-trimethylammonium-propane)-mediated transfection with $6.7 \mu \mathrm{g}$ siRNA was performed as described by incubating $10^{6} \mathrm{DCs}$ for $20 \mathrm{~h}$ at $37^{\circ} \mathrm{C} .{ }^{19} \mathrm{Cells}$ were then recovered, washed, and used immediately. Expression of IKK $\alpha$ and IKK $\beta$ transcripts in transfected or mocktransfected cells (i.e., cells treated with DOTAP alone) was evaluated by RT-PCR using specific primers. ${ }^{19}$

Enzyme-linked immunosorbent assay and reverse transcriptasePCR and real-time PCR. The levels of cytokines in colon homogenates and culture supernatants $(24 \mathrm{~h})$ of DCs or purified MLN CD4 ${ }^{+}$T cells from mice with colitis were determined by Kit enzyme-linked immunosorbent assay (ELISA) (R\&D Systems). Real-time RT-PCR was performed using the iCycler iQ detection system (Bio-Rad Laboratories, Life Science Group, Segrate, Italy) and SYBR Green chemistry (Finnzymes Oy, Espoo, Finland). Cells were lysed and total RNA was extracted using RNeasy Mini Kit (QIAGEN, Milan, Italy) and reversetranscribed with Sensiscript Reverse Transcriptase (QIAGEN) according to the manufacturer's directions. The PCR primers were as described ${ }^{2}$ or as follows: forward primer: $5^{\prime}$-CCCTATGGAGATGACGGAGA-3' and reverse primer: $5^{\prime}$-CTGTCTGCTGGTGGAGTTCA-3' for Ifnb1; 
forward primer: 5'-GGACAAGGAGGAAGAGGAAGAG-3' and reverse primer: 5' -CGAACAGCCCGAGACAGG-3' for IL-27p28; forward primer: $5^{\prime}$-ACCTTGACCTGTGCGGACTC-3' and reverse primer: 5'-GTTCGGCTTGGACCTCTGTTC-3' for Delta4; forward primer: $5^{\prime}$-GTGGCTTGGGTCTGTTGCTTGG-3' and reverse primer: 5'-CCGTGTTGGCTCCGTGTTTCTCG-3' for Jagged 1; forward primer: 5'-TGTGACGATGGCTGGAAGGG-3' and reverse primer: 5'-GCTGCTGTTCTTGGCGATGG-3' for Jagged 2. Amplification efficiencies were validated and normalized against Gapdh. The thermal profile for SYBR Green real-time PCR was at $95^{\circ} \mathrm{C}$ for $3 \mathrm{~min}$, followed by 40 cycles of denaturation for $30 \mathrm{~s}$ at $95^{\circ} \mathrm{C}$ and an annealing/extension step of $30 \mathrm{~s}$ at $60^{\circ} \mathrm{C}$. Each data point was examined for integrity by analysis of the amplification plot. The mRNA-normalized data were expressed as relative cytokine mRNA in stimulated cells compared with that of mock-infected cells.

Statistical analysis. Data were analyzed by GraphPad Prism 4.03 program (GraphPad Software, San Diego, CA). Student's $t$-test or analysis of variance (ANOVA) and Bonferroni's test were used to determine the statistical significance of differences in organ clearance and in vitro assays. Significance was defined as $P<0.05$. The data reported are either from one representative experiment out of three to five independent experiments (Western blotting and RT-PCR) or are otherwise pooled from three to five experiments. The in vivo groups consisted of six to eight mice per group.

SUPPLEMENTARY MATERIAL is linked to the online version of the paper at http://www.nature.com/mi

\section{ACKNOWLEDGMENTS}

We thank Dr Cristina Massi Benedetti for her editorial assistance. This study was supported by the Italian Projects PRIN 2007KLCKP8_004 (to LR) and 2007XYB9T9 (to SB) and Fondazione Cassa di Risparmio di Perugia Project n. 2006.020.0291 (Ricerce Scientifica).

\section{DISCLOSURE}

The authors declare no conflict of interest.

C 2009 Society for Mucosal Immunology

\section{REFERENCES}

1. Romani, L. \& Puccetti, P. Protective tolerance to fungi: the role of IL-10 and tryptophan catabolism. Trends Microbiol. 14, 183-189 (2006).

2. De Luca, A. et al. Functional yet balanced reactivity to Candida albicans requires TRIF, MyD88, and IDO-dependent inhibition of Rorc. J. Immunol. 179, 5999-6008 (2007).

3. Yoshitomi, H. et al. A role for fungal \{beta\}-glucans and their receptor Dectin- 1 in the induction of autoimmune arthritis in genetically susceptible mice. J. Exp. Med. 201, 949-960 (2005).

4. Romani, L., Bistoni, F. \& Puccetti, P. Fungi, dendritic cells and receptors: a host perspective of fungal virulence. Trends Microbiol. 10, 508-514 (2002).

5. Romani, L. et al. The exploitation of distinct recognition receptors in dendritic cells determines the full range of host immune relationships with Candida albicans. Int. Immunol. 16, 149-161 (2004).

6. Cambi, A. et al. Dendritic cell interaction with Candida albicans critically depends on N-linked mannan. J. Biol. Chem. 283, 20590-20599 (2008).

7. Filler, S.G. Candida-host cell receptor-ligand interactions. Curr. Opin. Microbiol. 9, 333-339 (2006).

8. Netea, M.G., Brown, G.D., Kullberg, B.J. \& Gow, N.A. An integrated model of the recognition of Candida albicans by the innate immune system. Nat. Rev. Microbiol. 6, 67-78 (2008).

9. Pulendran, B., Tang, H. \& Denning, T.L. Division of labor, plasticity, and crosstalk between dendritic cell subsets. Curr. Opin. Immunol. 20, 61-67 (2008).

10. Fallarino, F. et al. The combined effects of tryptophan starvation and tryptophan catabolites down-regulate $T$ cell receptor zeta-chain and induce a regulatory phenotype in naive T cells. J. Immunol. 176, 6752-6761 (2006)
11. Romani, L. et al. Thymosin alpha1 activates dendritic cell tryptophan catabolism and establishes a regulatory environment for balance of inflammation and tolerance. Blood 108, 2265-2274 (2006).

12. Montagnoli, C. et al. A role for antibodies in the generation of memory antifungal immunity. Eur. J. Immunol. 33, 1193-1204 (2003).

13. Pulendran, B. Variegation of the immune response with dendritic cells and pathogen recognition receptors. J. Immunol. 174, 2457-2465 (2005).

14. Naik, S.H. et al. Cutting edge: generation of splenic CD8+ and CD8dendritic cell equivalents in Fms-like tyrosine kinase 3 ligand bone marrow cultures. J. Immunol. 174, 6592-6597 (2005).

15. d'Ostiani, C.F. et al. Dendritic cells discriminate between yeasts and hyphae of the fungus Candida albicans. Implications for initiation of T helper cell immunity in vitro and in vivo. J. Exp. Med. 191, 1661-1674 (2000).

16. Shen, H., Tesar, B.M., Walker, W.E. \& Goldstein, D.R. Dual signaling of MyD88 and TRIF is critical for maximal TLR4-induced dendritic cell maturation. J. Immunol. 181, 1849-1858 (2008).

17. Ouaaz, F., Arron, J., Zheng, Y., Choi, Y. \& Beg, A.A. Dendritic cell development and survival require distinct NF-kappaB subunits. Immunity 16, 257-270 (2002).

18. Bonizzi, G. \& Karin, M. The two NF-kappaB activation pathways and their role in innate and adaptive immunity. Trends Immunol. 25, 280-288 (2004).

19. Grohmann, U. et al. Reverse signaling through GITR ligand enables dexamethasone to activate IDO in allergy. Nat. Med. 13, 579-586 (2007).

20. Tas, S.W. et al. Noncanonical NF-kappaB signaling in dendritic cells is required for indoleamine 2,3-dioxygenase (IDO) induction and immune regulation. Blood 110, 1540-1549 (2007).

21. Mellor, A.L. \& Munn, D.H. IDO expression by dendritic cells: tolerance and tryptophan catabolism. Nat. Rev. Immunol. 4, 762-774 (2004).

22. Puccetti, P. \& Grohmann, U. IDO and regulatory T cells: a role for reverse signalling and non-canonical NF-kappaB activation. Nat. Rev. Immunol. 7, 817-823 (2007).

23. Barton, B.E. STAT3: a potential therapeutic target in dendritic cells for the induction of transplant tolerance. Expert. Opin. Ther. Targets 10, 459-470 (2006).

24. Nadiminty, N., Lou, W., Lee, S.O., Lin, X., Trump, D.L. \& Gao, A.C. Stat3 activation of NF\{kappa\}B p100 processing involves CBP/p300-mediated acetylation. Proc. Natl Acad. Sci. USA 103, 7264-7269 (2006).

25. Puccetti, P. On watching the watchers: IDO and type I/II IFN. Eur. J. Immunol. 37, 876-879 (2007).

26. Goriely, S., Neurath, M.F. \& Goldman, M. How microorganisms tip the balance between interleukin-12 family members. Nat. Rev. Immunol. 8 , 81-86 (2008)

27. Amsen, D., Blander, J.M., Lee, G.R., Tanigaki, K., Honjo, T. \& Flavell, R.A. Instruction of distinct CD4 Thelper cell fates by different notch ligands on antigen-presenting cells. Cell 117, 515-526 (2004).

28. Veldhoen, M., Hocking, R.J., Flavell, R.A. \& Stockinger, B. Signals mediated by transforming growth factor-beta initiate autoimmune encephalomyelitis, but chronic inflammation is needed to sustain disease. Nat. Immunol. 7, 1151-1156 (2006).

29. Dillon, S. et al. Yeast zymosan, a stimulus for TLR2 and dectin-1, induces regulatory antigen-presenting cells and immunological tolerance. J. Clin. Invest. 116, 916-928 (2006).

30. Ma, C.S. et al. Deficiency of Th17 cells in hyper IgE syndrome due to mutations in STAT3. J. Exp. Med. 205, 1551-1557 (2008).

31. Milner, J.D. et al. Impaired T(H)17 cell differentiation in subjects with autosomal dominant hyper-lgE syndrome. Nature 452, 773-776 (2008).

32. Blaskovich, M.A., Sun, J., Cantor, A., Turkson, J., Jove, R. \& Sebti, S.M. Discovery of JSI-124 (cucurbitacin I), a selective Janus kinase/signal transducer and activator of transcription 3 signaling pathway inhibitor with potent antitumor activity against human and murine cancer cells in mice. Cancer Res. 63, 1270-1279 (2003).

33. Welte, T. et al. STAT3 deletion during hematopoiesis causes Crohn's disease-like pathogenesis and lethality: a critical role of STAT3 in innate immunity. Proc. Natl Acad. Sci. USA 100, 1879-1884 (2003).

34. Kobayashi, M. et al. Toll-like receptor-dependent production of IL-12p40 causes chronic enterocolitis in myeloid cell-specific Stat3-deficient mice. J. Clin. Invest. 111, 1297-1308 (2003).

35. Gurtner, G.J., Newberry, R.D., Schloemann, S.R., McDonald, K.G. \& Stenson, W.F. Inhibition of indoleamine 2,3-dioxygenase augments trinitrobenzene sulfonic acid colitis in mice. Gastroenterology 125 , 1762-1773 (2003). 


\section{ARTICLES}

36. Foligne, B. et al. A key role of dendritic cells in probiotic functionality. PLoS ONE 2, e313 (2007).

37. Slack, E.C. et al. Syk-dependent ERK activation regulates IL-2 and IL-10 production by DC stimulated with zymosan. Eur. J. Immunol. 37, 1600$1612(2007)$

38. Gringhuis, S.I. et al. Dectin-1 directs Thelper cell differentiation by controlling noncanonical NF-kappaB activation through Raf-1 and Syk. Nat. Immunol. 10, 203-213 (2009).

39. Rosas, M. et al. The induction of inflammation by dectin-1 in vivo is dependent on myeloid cell programming and the progression of phagocytosis. J. Immunol. 181, 3549-3557 (2008).

40. Caparros, E. et al. DC-SIGN ligation on dendritic cells results in ERK and PI3K activation and modulates cytokine production. Blood 107, 39503958 (2006).

41. Manicassamy, S. et al. Toll-like receptor 2-dependent induction of vitamin A-metabolizing enzymes in dendritic cells promotes T regulatory responses and inhibits autoimmunity. Nat. Med. 15, 401-409 (2009).

42. Bozza, S. et al. Lack of Toll IL1R8 exacerbates Th17 cell responses in fungal infection. J. Immunol. 180, 4022-4031 (2008).

43. Fukao, T. et al. PI3K-mediated negative feedback regulation of IL-12 production in DCs. Nat. Immunol. 3, 875-881 (2002).
44. Acosta-Rodriguez, E.V. et al. Surface phenotype and antigenic specificity of human interleukin 17-producing T helper memory cells. Nat. Immunol. 8, 639-646 (2007).

45. Leibundgut-Landmann, S. et al. Syk- and CARD9-dependent coupling of innate immunity to the induction of $\mathrm{T}$ helper cells that produce interleukin 17. Nat. Immunol. 8, 630-638 (2007).

46. Puig-Kroger, A. et al. Extracellular signal-regulated protein kinase signaling pathway negatively regulates the phenotypic and functional maturation of monocyte-derived human dendritic cells. Blood 98, 2175-2182 (2001).

47. Gerosa, F. et al. Differential regulation of interleukin 12 and interleukin 23 production in human dendritic cells. J. Exp. Med. 205, 1447-1461 (2008).

48. Beurel, E. \& Jope, R.S. Differential regulation of STAT family members by glycogen synthase kinase-3. J. Biol. Chem. 283, 21934-21944 (2008).

49. Harrington, L. et al. Deficiency of indoleamine 2,3-dioxygenase enhances commensal-induced antibody responses and protects against Citrobacter rodentium-induced colitis. Infect. Immun. 76, 3045-3053 (2008).

50. Pedersen, A.E., Gad, M., Kristensen, N.N., Haase, C., Nielsen, C.H. \& Claesson, M.H. Tolerogenic dendritic cells pulsed with enterobacterial extract suppress development of colitis in the severe combined immunodeficiency transfer model. Immunology 121, 526-532 (2007). 